\title{
Tools for the analysis and design of communication networks with Markovian dynamics
}

\author{
A. Leizarowitz, R. Stanojević and R. Shorten
}

\begin{abstract}
The stochastic properties of a class of communication networks whose dynamics are Markovian are analysed. The asymptotic behaviour of such a network in terms of the first and second moments of a stochastic process that describes the network dynamics is characterised and tools for their calculation are provided. Specifically, computation techniques for the calculation of these statistics are provided and that these algorithms converge exponentially fast is shown. Finally, how the results may be used for the design of network routers to realise networks with desired statistical properties is suggested.
\end{abstract}

\section{Introduction}

\subsection{General remarks}

The study of communication networks that carry transmission control protocol (TCP) traffic has been subject of intense interest in the computer science, network engineering, and applied mathematics literature [1-9].

The principal motivation for much of this work has been to understand network behaviour, and to characterise important network properties with a view to developing analytic tools for the design of such networks. In particular, much of this work has focussed on understanding the manner in which the network allocates available bandwidth among competing network flows (network fairness) and the speed at which this bandwidth allocation takes place (network convergence rate). Recently, a very accurate random matrix model of TCP network dynamics was proposed [10]. This model was shown to be capable of capturing many essential features of networks in which TCP-like network flows compete for bandwidth via a bottleneck router. By making some simplifying assumptions concerning stochastic behaviour of the network, the authors demonstrate that this model may also be used as a basis to design networks in which bandwidth can be allocated in an arbitrary manner among competing flows. This may be achieved by redesigning the manner in which individual sources respond to network congestion, or by redesigning the manner in which network routers respond to network congestion (or both).

The objective of this paper is to pursue further this line of research. However, rather than using the model as a basis for adjusting the behaviour of the individual flows to achieve desired network behaviour, we concentrate here on using this model to redesign the manner in which the bottleneck router drops packets when the network is congested;

(C) The Institution of Engineering and Technology 2006

IEE Proceedings online no. 20050171

doi:10.1049/ip-cta:20050171

Paper first received 10th May and in revised form 28th July 2005

A. Leizarowitz is with the Department of Mathematics, Technion, Israel

R. Stanojević and R. Shorten are with the Hamilton Institute, NUI Maynooth, Ireland

E-mail: la@techunix.technion.ac.il in particular, we analyse the properties of such networks when the bottleneck router drops packets according to some Markovian rules. Redesigning the manner in which network routers operate to allocate bandwidth is very important for a number of reasons related to network quality of service issues. Whereas the results in [10] are interesting from a theoretical perspective, router redesign along the lines suggested by this work would place an impossible computational burden on the network routers; on the other hand, dropping packets according to some Markovian rule could possibly be implemented using far fewer computational resources. Our principal contribution in this paper is to characterise the stochastic behaviour of these networks in terms of the first and second moments of a stochastic process that describes the network dynamics, and develop computational techniques for the calculation of these statistics. We concentrate on these statistics as they provide a characterisation of the average long-term fairness properties of network, and some measure of the instantaneous deviation (instantaneous unfairness) from this measure. Finally, we suggest how our results may be used to design new types of communication networks.

\subsection{Brief description of AIMD congestion control algorithms}

Most traffic in communication networks is carried using the TCP protocol $(85-90 \%$ of all Internet traffic is TCP-traffic [11]). The standard TCP protocol (introduced by [12]) is a special case of additive-increase multiplicative decrease (AIMD) congestion control. Here we give a very brief description of the AIMD congestion control strategy; the interested reader is referred to $[13,14]$ for a detailed description of the protocol.

A communication network consists of a number of sources and sinks connected together via links and routers. In this paper we assume that these links can be modelled as a constant propagation delay together with a queue, that the queue is operating according to a drop-tail discipline, and that all of the sources are operating an AIMD-like congestion control algorithm. AIMD congestion control operates a windowbased congestion control strategy. Each source maintains an internal variable $c w n d_{i}$ (the window size), which tracks the number of sent unacknowledged packets that can be in transit at any time, that is, the number of packets in flight. 
On safe receipt of data packets the destination sends acknowledgement (ACK) packets to inform the source. When the window size is exhausted, the source must wait for an ACK before sending a new packet. Congestion control is achieved by dynamically adapting the window size according to an AIMD law. Roughly speaking, the basic idea is for a source to probe the network for spare capacity by increasing the rate at which packets are inserted into the network, and to rapidly decrease the number of packets transmitted through the network when congestion is detected through the loss of data packets. In more detail, the source increments $c w n d_{i}(t)$ by a fixed amount $\alpha_{i}$ upon receipt of each ACK. On detecting packet loss, the variable $c w n d_{i}(t)$ is reduced in multiplicative fashion to $\beta_{i} c w n d_{i}(t)$. We shall see that the AIMD paradigm with drop-tail queuing gives rise to networks whose dynamics can be accurately modelled as a positive linear system.

\subsection{AIMD model and problem description}

Various types of models for AIMD networks have been developed by several authors; see [15] or [16] and the references therein for an overview of this work. We base our discussion on a recently developed random matrix model of AIMD dynamics that was first presented in [10]. This model uses a set of stochastic matrices to characterise the behaviour of a network of AIMD flows that compete for bandwidth via a single bottleneck router (as depicted in Fig. 1).

While other similar random matrix models have been proposed in the literature $[17,18]$, the model proposed in [10] has several attractive features. In particular, the authors use sets of non-negative column stochastic matrices to model the evolution of communication networks and results from Frobenius-Perron theory to characterise the stochastic properties of such networks. We begin our discussion by reviewing the essential features of this model.

Suppose that the network under consideration has $n$ flows, all of them operating an AIMD congestion control algorithm, competing for bandwidth over a bottleneck link which has drop-tail queue. Then the current state of the network at times when a packet is dropped at the bottleneck router (referred to as the $k$ th congestion event) is given by the number of packets in flight that belong to each network source at this time. We describe the network state at the $k$ th congestion event by an $n$-dimensional vector $W(k)=\left\{w_{i}(k)\right\}_{i=1}^{n}$ where $w_{i}(k)$ is the $i$ th component of $W(k)$, which is equal to the throughput that belongs to the $i$ th source when this source is informed of network congestion. It has been shown in [10] that the sequence

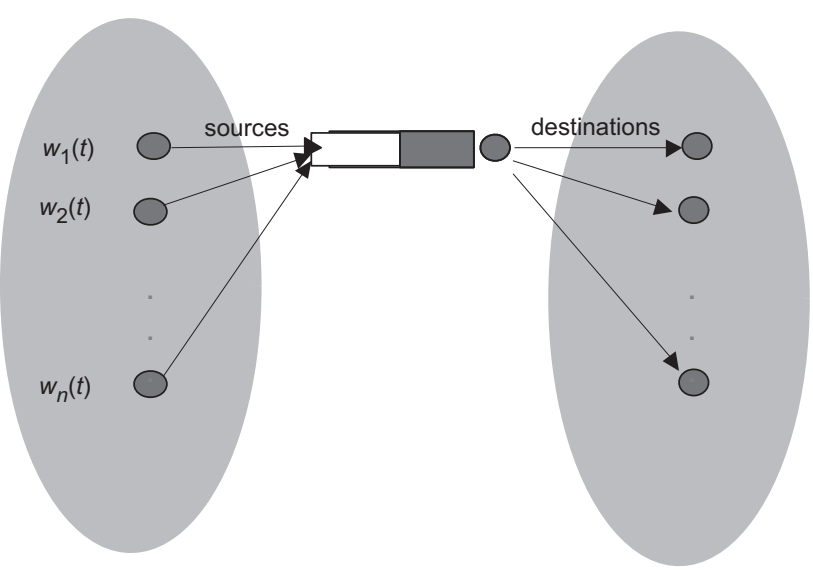

Fig. 1 Network with single bottleneck router
$\{W(k)\}_{k=0}^{\infty}$ satisfies

$$
W(k+1)=A(k) W(k)
$$

where $W(k)=\left[w_{1}(k), \ldots, w_{n}(k)\right]^{T}$, and

$$
\begin{aligned}
A(k)= & {\left[\begin{array}{cccc}
\beta_{1}(k) & 0 & \cdots & 0 \\
0 & \beta_{2}(k) & 0 & 0 \\
\vdots & 0 & \ddots & 0 \\
0 & 0 & \cdots & \beta_{n}(k)
\end{array}\right] } \\
& +\frac{1}{\sum_{j=1}^{n} \alpha_{j} \gamma_{j}}\left[\begin{array}{c}
\alpha_{1} \gamma_{1} \\
\alpha_{2} \gamma_{2} \\
\cdots \\
\alpha_{n} \gamma_{n}
\end{array}\right]\left[1-\beta_{1}(k), \ldots, 1-\beta_{n}(k)\right]
\end{aligned}
$$

For every $j \in\{1,2, \ldots, n\}$, the constant $\alpha_{j}>0$ in (2) is the additive increase parameter and $\gamma_{j}>0$ is the constant $1 / R T T_{j}^{2}$. Here $R T T_{j}$ is the round-trip time for a packet from the $j$ th flow just before congestion, and either $\beta_{j}(k)=1$, which holds if the $j$ th flow did not lose any packet during the $k$ th congestion event, or $\beta_{j}(k)$ is equal to the multiplicative decrease parameter $\beta_{j}^{0} \in(0,1)$ if the $j$ th flow did lose some packet in the $k$ th congestion event.

Comment: We exclude the possibility that $\beta_{1}(k)=$ $\beta_{2}(k)=\cdots=\beta_{n}(k)=1$, since there is no congestion event without losing at least one packet.

We denote by $\mathcal{M}$ the set of the possible values of the matrices $A(k)$, so that

$$
\mathcal{M}=\left\{M_{1}, M_{2}, \ldots, M_{m}\right\}
$$

for some $m \leq 2^{n}-1$ and for all $k$. Then $A(k) \in \mathcal{M}$ for every $k \geq 0$, and we note that the strict inequality $m<2^{n}-1$ may hold; namely that in the models which we consider, certain configurations of packets' loss cannot practically occur.

Let $I(k)=\left\{j: \beta_{j}(k)=\beta_{j}^{0}\right\}$ be the set of labels of flows that have experienced a loss of a packet during the $k$ th congestion event. Note that for each $k$ the matrix $A(k)$ has a strictly positive $j$ th column if and only if $j \in I(k)$, and that for $j \notin$ $I(k)$, the $j$ th column of $A(k)$ is equal to $e_{j}$, the $j$ th column of the identity $n \times n$ matrix $I_{n}$. We denote by $\Sigma$ the $(n-1)$ dimensional simplex of all the $n$-dimensional stochastic vectors. Recall that a vector $v=\left(v_{1}, \ldots, v_{n}\right) \in \mathbb{R}^{n}$ is stochastic if each one of its coordinates $v_{i}$ is nonnegative and $v_{1}+\cdots+v_{n}=1$. It turns out that the matrices $M_{i}$ $(1 \leq i \leq m)$ that compose $\mathcal{M}$, are non-negative and column-stochastic [19]. Therefore, $M_{i}(\Sigma) \subset \Sigma$ holds for every $1 \leq i \leq m$. By normalising $W(0)$ to belong to $\Sigma$, we may therefore assume, with no loss of generality, that $W(k) \in \Sigma$ for every $k \geq 0$.

For networks with routers employing a drop-tail queueing discipline, it is often assumed in the networking community that congestion events may in some circumstances be modelled as sequences of independent events $[10,17,20,21]$. In terms of the model described above, this means that for networks with a single bottleneck link and with a drop-tail queue the following assumption holds.

Assumption 1: $\{A(k)\}_{k \in N}$ is a sequence of independent and identically distributed (i.i.d.) random variables $A(k)$, and 
for every $j \in\{1,2, \ldots, n\}$ the probability that the $j$ th flow detects a drop in each congestion event is positive.

As we have mentioned already, in designing rules that determine how the network will react to congestion, one can typically have two approaches. The first is the design of flow-based congestion control algorithms and the second is the design of queueing discipline. In the present paper we concentrate on the latter and propose two new queueing disciplines, which we characterise by computing the stationary statistics for the vector $W(k)$ for each of these cases. We first consider a queuing discipline with the property that packets are dropped from the queue in such a manner that the following assumption is valid.

Assumption 2: $\{A(k)\}_{k \in N}$ is a stationary Markov chain on the finite set of matrices $\mathcal{M}$ with transition matrix $P \in \mathbb{R}^{m \times m}$. Moreover, we assume that for each $j \in\{1,2, \ldots, n\}$ there exists a matrix $M \in \mathcal{M}$ with positive jth column.

We note that as in the i.i.d. case, we must have the latter assumption in order to ensure that each flow will see a drop at some point. We also remark that stationarity is assumed to avoid technical difficulties and it is not essential. An additional assumption in Sections 2 and 3 (which is relaxed in Section 4 ) is that the transition matrix $P$ has strictly positive entries. Theorems 2 and 4 give the asymptotic values of $E(W(k))$ and $E\left[(W(k))(W(k))^{T}\right]$ in the limit where $k$ tends to infinity, which we denote $V^{*}$ and $D^{*}$, respectively. Although we do not have explicit formulas for $V^{*}$ and $D^{*}$, theorems 1 and 3 provide iterative algorithms for computing them in a geometric convergence rate. In Section 4 we extend the results of the previous two sections to the case where the matrix $P$ is merely primitive and its entries are not necessarily strictly positive.

The second queueing discipline we propose here is the following: the probability that a certain set of flows will detect a drop during the $k$ th congestion event depends only on the vector $W(k)$. Formally we assume that the router drops a packet from the queue when it is full in such a fashion that the following assumption is true.

Assumption 3: $\{W(k)\}_{k \in N}$ is a stochastic process in the set of stochastic vectors $\Sigma$, which has the following property:

For every $i \in\{1,2, \ldots, m\}$ and $w \in \Sigma$, the conditional probability of $A(k)$ given $W(k)$ is expressed by

$$
P\left[A(k)=M_{i} \mid W(k)=w\right]=p_{i}(w)
$$

for some positive continuous functions $p_{i}: \Sigma \rightarrow \mathbb{R}^{+}$, which satisfy $\sum_{i=1}^{m} p_{i}(w)=1$ for all $w \in \Sigma$. Again, for each $i \in\{1,2, \ldots, n\}$ we require the existence of a matrix $M \in \mathcal{M}$ with positive $i$ th column.

In view of the relation $W(k+1)=A(k) W(k)$, assumption 3 implies that the distribution of $W(k+1)$ is completely determined by the distribution of $W(k)$. Section 5 is devoted to studying the behaviour of $W(k)$ under assumption 3 . It turns out that the study of the model under assumption 3 can be reduced to its study under assumption 2 . This enables to establish the analogous results concerning the asymptotic behaviour of $E(W(k))$ and $\operatorname{Var}(W(k))$ for this case. In particular, the latter can be computed by iterative methods producing schemes that converge in a geometric rate.

\section{The asymptotic expectation of $W(N)$}

In this Section we compute the equilibrium expected value of the window size variable $W(N)$ under assumption 2 , and supposing that the transition probabilities $P_{i j}$ are positive:

$$
P_{i j}>0 \quad \text { for every } 1 \leq i, j \leq m
$$

Denoting by $\rho=\left(\rho_{1}, \ldots, \rho_{m}\right)$ the unique equilibrium distribution corresponding to $P$, we associate with $P_{i j}$ the backward transition probabilities matrix $\tilde{P}$ (see [22], Chapter 1.9) given by

$$
\begin{aligned}
\tilde{P}_{i j}= & \frac{\rho_{i}}{\rho_{j}} P_{i j}=\frac{P\left[A(k-1)=M_{i}\right]}{P\left[A(k)=M_{j}\right]} \\
& \times P\left[A(k)=M_{j} \mid A(k-1)=M_{i}\right] \\
= & P\left[A(k-1)=M_{i} \mid A(k)=M_{j}\right]
\end{aligned}
$$

We interpret $\tilde{P}_{i j}$ as the conditional probability that the system occupied the state $M_{i}$ at the previous instant of time given that it is presently at state $M_{j}$, for the stationary Markov chain $\left\{A_{k}\right\}$.

Let $\Phi:\left(\mathbb{R}^{n}\right)^{m} \rightarrow\left(\mathbb{R}^{n}\right)^{m}$ be the linear mapping given by

$$
\Phi(V)=\left(\sum_{i=1}^{m} \tilde{P}_{i 1} M_{i} V_{i}, \ldots, \sum_{i=1}^{m} \tilde{P}_{i m} M_{i} V_{i}\right)
$$

where $V=\left(V_{1}, \ldots, V_{m}\right), V_{i} \in \mathbb{R}^{n}$ and $M_{i} \in \mathcal{M}$ for every $1 \leq i \leq m$. We have the following result:

Proposition 1: For an arbitrary $W(0)=s \in \Sigma$ and all $i=1$, $2, \ldots, m$, the following limits exist:

$$
V_{i}=\lim _{k \rightarrow \infty} E\left[W(k) \mid A(k)=M_{i}\right], \quad i=1,2, \ldots, m
$$

Moreover, the vector $V=\left(V_{1}, \ldots, V_{m}\right) \in \Sigma^{m}$ whose components are defined in (6) satisfies the fixed point equation

$$
V=\Phi(V)
$$

Proof: The proof will be given after establishing Theorem 2.7.

Let $\mathcal{S}$ be the subspace of $\mathbb{R}^{n}$ defined by

$$
\mathcal{S}=\left\{x \in \mathbb{R}^{n}: \sum_{i=1}^{n} x_{i}=0\right\}
$$

It turns out that $\Phi$ has the following property:

Proposition 2: The mapping $\Phi$ is linear from $\Sigma^{m}$ into itself, and from $\mathcal{S}^{m}$ into itself.

Proof: Both claims follow from the facts that if $M$ is a column stochastic $n \times n$ matrix, then for every $x \in \mathbb{R}^{n}$ we have

$$
\sum_{i=1}^{n}(M x)_{i}=\sum_{i=1}^{n} x_{i}
$$

and each $M_{i}$ is a column stochastic matrix.

We know that any matrix in $\mathcal{M}$ can be written in the form

$$
\begin{aligned}
M= & \operatorname{diag}\left(\beta_{1}, \beta_{2}, \ldots, \beta_{n}\right) \\
& +\left(\delta_{1}, \ldots, \delta_{n}\right)^{T}\left(\left(1-\beta_{1}\right), \ldots,\left(1-\beta_{n}\right)\right)
\end{aligned}
$$

where $0<\beta_{k} \leq 1$ for every $1 \leq k \leq n$ and not all of them are equal to 1 . We denote $\bar{\beta}=\left(\beta_{1}, \ldots, \beta_{n}\right)^{T}$, 
$\delta=\left(\delta_{1}, \ldots, \delta_{n}\right)^{T}$, and $\delta$ is a stochastic vector with positive entries. If the first $q$ entries of $\beta$ are equal to 1 , namely $\beta_{1}=\beta_{2}=\ldots=\beta_{q}=1$ for some $q<n$, and the last $n-q=r>0$ entries are all smaller than 1 , then our matrix $M$ has the following form

$$
M=\left(\begin{array}{cc}
I_{q} & M^{\prime} \\
0 & M^{\prime \prime}
\end{array}\right)
$$

The matrix $I_{q}$ in (8) is the $q \times q$ identity matrix; all the entries in the last $r$ columns are positive, and the sum of the entries in each of these columns is equal to 1 .

The following result is the main technical tool that we employ in studying properties of $\Phi$. We denote by $\|\cdot\|_{1}$ the $L_{1}$ norm of vectors in $\mathbb{R}^{n}$. We will prove it as corollary 2 after having established lemma 3. A direct proof can be found in [23].

Lemma 1: Let $M \in \mathcal{M}$. Then for every $x \in \mathcal{S}$ we have

$$
M x \neq x \Longrightarrow\|M x\|_{1}<\|x\|_{1}
$$

Proof: See the proof of corollary 2.

Remark 1: The property that is established in lemma 1 is referred to in the literature as the paracontracting property; see [24] chapter 8 or [25]. We have thus shown that the matrices $M_{i}$ are paracontractive in $\mathcal{S}$ in $L_{1}$ norm. We will again use the notion of paracontractivity in Section 5 .

Lemma 2: Suppose that $M \in \mathcal{M}$ is such that the columns that contain zeros are indexed by $i_{1}, i_{2}, \ldots, i_{q}$, and let $x \in \mathcal{S}$ be such that $M x=x$. Then $x$ belongs to the subspace spanned by the basic vectors $e_{i_{1}}, e_{i_{2}}, \ldots, e_{i_{q}}$.

Proof: We suppose without loss of generality that the first $q$ columns of $M$ contain zeros and the last $r=n-q$ columns are positive, that is, that $M$ has the form given by (8). We will establish that the last $r$ coordinates of $x$ are equal to 0 . If $r=0$ then there is nothing to prove. If $r=n$ then $M$ is a stochastic matrix with strictly positive entries, and therefore it is a contraction on $\mathcal{S}$, implying that $x=0$.

We now suppose that $0<r<n$, and let $P$ be the $r \times r$ submatrix of $M$, which is defined by the last $r$ rows and last $r$ columns. If we denote by $x^{\prime}$ the $r$-dimensional vector composed of the last $r$ coordinates of $x$, then $P x^{\prime}=x^{\prime}$. But the sum of each column of $P$ is smaller than 1 and $x$ is non-negative, hence $\left\|P x^{\prime}\right\|_{1}<\left\|x^{\prime}\right\|_{1}$ whenever $x^{\prime} \neq 0$, implying that $x^{\prime}$ must vanish. This concludes the proof of the lemma.

We define on $\left(\mathbb{R}^{n}\right)^{m}$ the norm

$$
\|V\|=\left\|\left(V_{1}, \ldots, V_{m}\right)\right\|=\max _{1 \leq i \leq m}\left(\left\|V_{i}\right\|_{1}\right)
$$

and consider the subspace $\mathcal{S}^{m}$ and subset $\Sigma^{m}$ of $\left(\mathbb{R}^{n}\right)^{m}$ endowed with this norm. The next result establishes that $\Phi^{2}$ is a contraction on the metric space $\Sigma^{m}$ as well as on normed space $\mathcal{S}^{m}$.

Proposition 3: Let $\Phi$ be the mapping given by (5). We assume that (3) holds, so that in view of (4), all the backward probabilities $\tilde{P}_{i j}$ are positive as well. Then there exists a constant $\theta<1$ such that

$$
\left\|\Phi^{2}(U)-\Phi^{2}(V)\right\| \leq \theta\|U-V\|
$$

holds for all $U, V \in \Sigma$.
Proof: We will establish that for every pair $U \neq V$ in $\Sigma$, the inequality $\left\|\Phi^{2}(U)-\Phi^{2}(V)\right\|<\|U-V\|$ holds. This will imply the assertion of the proposition in view of the compactness of $\Sigma^{m}$.

Thus let $U=\left(U_{1}, \ldots, U_{m}\right)$ and $V=\left(V_{1}, \ldots, V_{m}\right)$ be any two different elements belonging to $\Sigma^{m}$. We have

$$
\begin{aligned}
\|\Phi(U)-\Phi(V)\| & =\max _{j}\left\|\sum_{i=1}^{m} \tilde{P}_{i j} M_{i}\left(U_{i}-V_{i}\right)\right\|_{1} \\
& \leq \max _{j} \sum_{i=1}^{m} \tilde{P}_{i j}\left\|M_{i}\left(U_{i}-V_{i}\right)\right\|_{1} \\
& \leq \max _{j} \sum_{i=1}^{m} \tilde{P}_{i j}\left\|U_{i}-V_{i}\right\|_{1} \\
& \leq \max _{j} \sum_{i=1}^{m} \tilde{P}_{i j}\|U-V\|=\|U-V\|
\end{aligned}
$$

We will next check under which conditions equality $\left\|\Phi^{2}(U)-\Phi^{2}(V)\right\|=\|U-V\|$ can hold. We thus assume that $U \neq V$ are such that $\| \Phi(\Phi(U))-\Phi(\Phi(V))=$ $\|U-V\|$. It follows from

$$
\begin{aligned}
\|U-V\| & =\|\Phi(\Phi(U))-\Phi(\Phi(V))\| \\
& \leq\|\Phi(U)-\Phi(V)\| \leq\|U-V\|
\end{aligned}
$$

that $\|\Phi(U)-\Phi(V)\|=\|U-V\|$. Thus, in this situation, all the inequalities in (11)-(14) are actually equalities.

We now denote $W=U-V \in \mathcal{S}^{m}$ and we note that in view of (14), for some $j$,

$$
\sum_{i=1}^{m} \tilde{P}_{i j}\left\|W_{i}\right\|_{1}=\max _{i}\left(\left\|W_{i}\right\|_{1}\right)=\|W\|
$$

Since we suppose that all $\tilde{P}_{i j}$ are positive, (15) implies

$$
\left\|W_{i}\right\|_{1}=\|W\| \quad \text { for every } 1 \leq i \leq m
$$

It then follows from (13) that

$$
\max _{j} \sum_{i=1}^{m} \tilde{P}_{i j}\left\|M_{i}\left(W_{i}\right)\right\|_{1}=\max _{j} \sum_{i=1}^{m} \tilde{P}_{i j}\left\|W_{i}\right\|_{1}=\|W\|
$$

which in view of $\left\|M_{i}\left(W_{i}\right)\right\|_{1} \leq\left\|W_{i}\right\|_{1}$ and the positivity of all the $P_{i j}$, implies

$$
\left\|M_{i}\left(W_{i}\right)\right\|_{1}=\|W\|
$$

for all $i$. It follows from (16), (17) and lemma 1 that

$$
M_{i}\left(W_{i}\right)=W_{i}
$$

for all $i$. We thus conclude from (12), (17) and (18) that there exist some $j$ such that

$$
\left\|\sum_{i=1}^{m} \tilde{P}_{i j}\left(W_{i}\right)\right\|_{1}=\sum_{i=1}^{m} \tilde{P}_{i j}\left\|W_{i}\right\|_{1}=\|W\|
$$

However, this can happen if and only if for every $r \in$ $\{1,2, \ldots, n\}$, there does not exist $1 \leq i, j \leq m$ such that the $r$ th coordinates $\left(W_{i}\right)_{r}$ and $\left(W_{j}\right)_{r}$ are of opposite signs.

By employing the above argument, and also the conclusion (18) to the equality $\|\Phi(\Phi(W))\|=\|\Phi(W)\|$ rather than to $\|\Phi(W)\|=\|W\|$, we have that for all $k \in\{1,2, \ldots, m\}$

$$
M_{k}\left(\sum_{i=1}^{m} \tilde{P}_{i k} W_{i}\right)=\sum_{i=1}^{m} \tilde{P}_{i k} W_{i}
$$


Assumption 2 of our model is such that for every $r \in\{1,2, \ldots, n\}$ there exists a matrix $M_{k} \in \mathcal{M}$ with positive $r$ th column. It follows from lemma 2 and (19) that the $r$ th coordinate of $\sum_{i=1}^{m} \tilde{P}_{i k} W_{i}$ must vanish. But there are no two indices $i_{1}$ and $i_{2}$ such that the $r$ th coordinates of $W_{i_{1}}$ and $W_{i_{2}}$ have opposite signs. This fact implies that the $r$ th coordinate of the vector $W_{i}$ must vanish, and this is true for every $1 \leq i \leq m$. Because $r$ is arbitrary, we conclude that $W_{i}=0$ for all $i$. We have thus established that if $U, V \in \Sigma^{m}$ are distinct then

$$
\left\|\Phi^{2}(U)-\Phi^{2}(V)\right\|<\|U-V\|
$$

The proof of the proposition is thus complete.

Theorem 1: The spectral radius of the restriction of $\Phi$ to $\mathcal{S}^{m}$ is smaller than 1. In particular there exists a unique solution $V^{*}$ for (7), and the iteration scheme

$$
V^{(k+1)}=\Phi\left(V^{(k)}\right), \quad k=0,1,2, \ldots
$$

with any starting point $V^{(0)}=V_{0}$ in $\Sigma$ satisfies

$$
\lim _{k \rightarrow \infty} V^{(k)}=V^{*}
$$

Proof: We will first establish that the spectral radius of the restriction of $\Phi$ to $\mathcal{S}^{m}$ is smaller than 1 . But we know that the iterations of $\Phi^{2}$ in $\mathcal{S}^{m}$ converge to zero for every starting point. It follows that every eigenvalue of the restriction of $\Phi$ to $\mathcal{S}^{m}$, say $\lambda$, satisfies $|\lambda| \leq 1$. If, however, there exists an eigenvalue that is equal to $e^{i \theta}$ for some real $\theta$, then there exists a subspace $\Pi$ of $\mathcal{S}^{m}$, which is invariant under $\Phi$, and is either one- or two-dimensional. The restriction of $\Phi$ to $\Pi$ is then a rotation, contradicting the fact that the iterations of $\Phi$ should tend to zero.

The uniqueness of solutions of theorem 1 follows from the contractive property of $\Phi^{2}$. Then, for every initial $V_{0} \in \Sigma$ we have $\lim _{k \rightarrow \infty} V^{(2 k)}=V^{*}$. Hence

$$
V^{(2 k+1)}=\Phi\left(V^{(2 k)}\right) \longrightarrow \Phi\left(V^{*}\right)=V^{*} \quad \text { as } k \longrightarrow \infty
$$

and (20) follows.

Proof of Proposition 1: Let $V_{i}(k)=E\left[W(k) \mid A(k)=M_{i}\right]$. The sequence of vectors $V(k)=\left(V_{1}(k), \ldots, V_{m}(k)\right) \in \Sigma^{m}$ satisfies

$$
V(k+1)=\Phi(V(k))
$$

From theorem $1,\{V(k)\}_{k=0}^{\infty}$ converge and the existence of the limits in (6) follows. In view of (21), these limits satisfy the fixed point equation (7).

We have the following result, which is actually theorem 3.1 from [10].

Corollary 1: Let assumption (1) hold, so that the probability that $A(k)=M_{i}$ is equal to $\rho_{i}$ for every $k \geq 0$ and $1 \leq i \leq m$. Then the asymptotic expected value of $W(k)$ is the unique stochastic eigenvector of $\sum_{i=1}^{m} \rho_{i} M_{i}$, which corresponds to the eigenvalue 1 .

Proof: The sequence $\{A(k)\}$ of i.i.d. random matrices can be seen as a Markov chain on the set $\mathcal{M}=\left\{M_{i}: \rho_{i}>0\right\}$ with the $m \times m$ transition matrix $P$ given by $P_{i j}=\rho_{j}$. Since $P_{i j}$ is positive for every $i$ and $j$, we have that $\tilde{P}_{i j}=$ $\rho_{i} P_{i j} / \rho_{j}=\rho_{i}>0$. We look for a solution of (1) for which all the components $V_{i}$ are the same, say equal to $\bar{V}$. This yields the equation

$$
\bar{V}=\left(\sum_{i=1}^{m} \rho_{i} M_{i}\right) \bar{V}
$$

which implies the assertion of the corollary.

Theorem 2: Under assumption 2, and assuming that the transition matrix $P$ has strictly positive entries, then the asymptotic behaviour of the expectation of the random variable $W(N)$ is given by

$$
\lim _{N \rightarrow \infty} E(W(N))=\sum_{i=1}^{m} \rho_{i} V_{i}^{*}
$$

where $V^{*}=\left(V_{1}^{*}, \ldots, V_{m}^{*}\right) \in \Sigma^{m}$ is the unique solution of (7), and $\rho=\left(\rho_{1}, \ldots, \rho_{m}\right)$ is the Perron eigenvector of the transition probability matrix $\left(P_{i j}\right)$.

Proof: The proof is immediate

$$
\begin{aligned}
\lim _{N \rightarrow \infty} E(W(N)) \\
=\lim _{N \rightarrow \infty} \sum_{i=1}^{m} E\left[W(N) \mid A(N)=M_{i}\right] P\left[A(N)=M_{i}\right] \\
=\sum_{i=1}^{m} \rho_{i} V_{i}^{*}
\end{aligned}
$$

\section{The asymptotic variance of $W(N)$}

The goal of this section is to compute the asymptotic value of the second order moment of $W(N)$ under assumption 2 and assuming a positive transition matrix $P$. (What we call variance, or second order moment is actually covariance matrix for the vector $W(N): E\left[(W(N))_{i}\left((W(N))_{j}\right)\right]-$ $E\left[(W(N))_{i}\right] E\left[(W(N))_{j}\right]$.)

Define the linear mapping $\Psi:\left(\mathbb{R}^{n \times n}\right)^{m} \rightarrow\left(\mathbb{R}^{n \times n}\right)^{m}$ by:

$$
\Psi\left(D_{1}, \ldots, D_{m}\right)=\left(\sum_{i=1}^{m} \tilde{P}_{i 1} M_{i} D_{i} M_{i}^{T}, \ldots, \sum_{i=1}^{m} \tilde{P}_{i m} M_{i} D_{i} M_{i}^{T}\right)
$$

Suppose for a moment that for $W(0)=s \in \Sigma$ the following limits exist:

$$
D_{i}=\lim _{k \rightarrow \infty} E\left[W(k) W(k)^{T} \mid A(k)=M_{i}\right]
$$

Comment: Note that each $D_{i}$ must be a symmetric nonnegative definite matrix, that it has non-negative entries and it satisfies

$$
\begin{aligned}
D_{i} u & =\lim _{k \rightarrow \infty} E\left[W(k) W(k)^{T} u \mid A(k)=M_{i}\right] \\
& =\lim _{k \rightarrow \infty} E\left[W(k) \mid A(k)=M_{i}\right]=V_{i}
\end{aligned}
$$

where $u$ is the $n$-dimensional vector that satisfies $u_{i}=1$ for every $1 \leq i \leq n$.

In view of (24) let $\mathcal{D}$ be the set

$$
\mathcal{D}=\left\{\left(D_{1}, \ldots, D_{m}\right) \mid D_{i} \in \mathbb{R}^{n \times n}, D_{i}=D_{i}^{T}, D_{i} u=V_{i}\right\}
$$

It turns out that $\Psi$ maps $\mathcal{D}$ into itself. Indeed, $(\Psi(D))_{j}$ is symmetric whenever all $D_{i}$ are such. Moreover, using (7) 
we obtain

$$
\sum_{i=1}^{m} \tilde{P}_{i j} M_{i} D_{i} M_{i}^{T} u=\sum_{i=1}^{m} \tilde{P}_{i j} M_{i} D_{i} u=\sum_{i=1}^{m} \tilde{P}_{i j} M_{i} V_{i}=V_{j}
$$

implying that $\Psi(D) \in \mathcal{D}$ for every $D \in \mathcal{D}$. We have the following result.

Proposition 4: For arbitrary $W(0)=s \in \Sigma$ and all $i \in$ $\{1,2, \ldots, m\}$ the following limits exist

$$
D_{i}=\lim _{k \rightarrow \infty} E\left[W(k) W(k)^{T} \mid A(k)=M_{i}\right]
$$

The $m$-tuple $D=\left(D_{1}, \ldots, D_{m}\right) \in \mathcal{D}$ defined by (26) satisfies the fixed point equation

$$
D=\Psi(D)
$$

Proof: The proof will be given after having established theorem (3).

Let

$$
\mathcal{B}=\left\{C \mid C \in \mathbb{R}^{n \times n}, C=C^{T}, C u=0\right\}
$$

Thus $\mathcal{B}$ is the vector space of all $n \times n$ symmetric matrices $C$ such that all the columns of $C$ belong to $\mathcal{S}$. A computation similar to the one preceding proposition 4 implies that $\Psi\left(\mathcal{B}^{m}\right) \subset \mathcal{B}^{m}$. Since the difference between any two elements from $\mathcal{D}$ belongs to $\mathcal{B}^{m}$, then fixing any norm on $\left(\mathbb{R}^{n \times n}\right)^{m}$, it follows that the linear mapping $\Psi$ is a contraction on the metric space $\mathcal{D}$ if it is a contraction on the vector space $\mathcal{B}^{m}$. We wish to establish the existence and uniqueness of solutions $D \in \mathcal{D}$ of (27). To this end, it is enough to find a norm in which the mapping $\Psi^{2}$ is a contraction on the complete metric space $\mathcal{D}$.

Let $\|\cdot\|$ be the norm on $\mathbb{R}^{n \times n}$ defined by

$$
\|A\|=\sum_{i, j=1}^{n}\left|A_{i j}\right| \quad \text { for } A \in \mathbb{R}^{n \times n}
$$

The next result establishes a crucial connection between this norm and the mapping $C \mapsto M C M^{T}$ for $C \in \mathcal{B}$ and $M \in \mathcal{M}$. It is close in spirit to lemma 1.

Lemma 3: Let $M \in \mathcal{M}$. Then the following relation

$$
M C \neq C \Longrightarrow\left\|M C M^{T}\right\|<\|C\|
$$

holds for every $C \in \mathcal{B}$.

Proof: As in the proof of lemma 2, we consider a matrix $M$ that has the form (8) for some $0 \leq q<n$, and where the last $r=n-q$ columns are positive.

We then have

$$
\begin{aligned}
\left\|M C M^{T}\right\| & =\sum_{i, j}\left|\sum_{k, l} m_{i k} c_{k l} m_{j l}\right| \leq \sum_{i, j} \sum_{k, l} m_{i k} m_{j l}\left|c_{k l}\right| \\
& =\sum_{k, l}\left|c_{k l}\right| \sum_{i} m_{i k} \sum_{j} m_{j l}=\sum_{k, l}\left|c_{k l}\right|=\|C\|
\end{aligned}
$$

since $M$ is column stochastic. We have thus established

$$
\left\|M C M^{T}\right\| \leq\|C\|
$$

for any column stochastic matrix $M$ and any matrix $C$. We will next prove that equality holds in (31) only if $M C=C$.
We remark that if $M C=C$, then in view of $C=C^{T}$, we have

$$
M C M^{T}=C M^{T}=(M C)^{T}=C^{T}=C
$$

implying that equality holds in (31) if $M C=C$.

We now suppose that $M$ and $C$ are such that $\left\|M C M^{T}\right\|=\|C\|$. This is possible if and only if for each pair $1 \leq i, j \leq n$, the only inequality that appears in (30) is actually an equality. This, however, holds if and only if for every $1 \leq i, j \leq n$ the following holds:

Property S: There are no two pairs of indices $(k, l)$ and $\left(k^{\prime}, l^{\prime}\right)$ such that $m_{i k} m_{j l}$ and $m_{i k^{\prime}} m_{j l^{\prime}}$ are both positive while $c_{k l}$ and $c_{k^{\prime} l^{\prime}}$ have opposite signs.

For $1 \leq i \leq q$ let $\Lambda_{i}=\left\{c_{i l} \mid q<l \leq n\right\}=\left\{c_{l i} \mid q<l \leq n\right\}$, and denote $\Lambda_{0}=\left\{c_{k l} \mid q<k \leq n, \quad q<l \leq n\right\}$. Using property $\mathrm{S}$ for a pair $i, j \in\{1,2, \ldots, q\}$, and noting that for all $k, l \in\{q+1, q+2, \ldots, n\}$, we have $m_{i k} m_{j j}>0$, $m_{i i} m_{j l}>0$ and $m_{i k} m_{j l}>0$, and further we conclude that there are no two elements in set $\Lambda_{i j}=\Lambda_{i} \cup \Lambda_{j} \cup \Lambda_{0}$ with opposite sign. Since for each pair of indices $(k, l)$ and $\left(k^{\prime}, l^{\prime}\right)$ with $\max \{k, l\}>q$ and $\max \left\{k^{\prime}, l^{\prime}\right\}>q$ there is pair $i, j \in\{1,2, \ldots, q\}$ such that both $c_{k l}$ and $c_{k^{\prime} l^{\prime}}$ are contained in $\Lambda_{i j}$, we conclude that either

$$
c_{k l} \geq 0 \quad \text { whenever } \max \{k, l\}>q
$$

or

$$
c_{k l} \leq 0 \quad \text { whenever } \max \{k, l\}>q
$$

For any integer $q<l \leq n$, the sum of the entries in the $l$ th column (or row) have the same sign as the constant sign of its elements, namely non-negative (non-positive) if (32) [(33)] holds. Since this sum is zero, we conclude that all the entries $c_{k l}$ are such that at least one of $k>q$ and $l>q$ holds must vanish. Thus $C$ must have the form

$$
C=\left(\begin{array}{cc}
C^{\prime} & 0 \\
0 & 0
\end{array}\right)
$$

where $C^{\prime} \in \mathbb{R}^{q \times q}$, and since

$$
M=\left(\begin{array}{cc}
I_{q} & M^{\prime} \\
0 & M^{\prime \prime}
\end{array}\right)
$$

it follows that $M C=C$, concluding the proof of the lemma.

Now we are able to present the following short proof of lemma 1.

Corollary 2: Let $M \in \mathcal{M}$. Then for every $x \in \mathcal{S}$ we have

$$
M x \neq x \Longrightarrow\|M x\|_{1}<\|x\|_{1}
$$

Proof: Note that for $x \in S, C=x x^{T} \in \mathcal{B}$ we can conclude that

$$
\left\|M C M^{T}\right\|=\left\|M x x^{T} M^{T}\right\|=\|M x\|_{1}^{2} \leq\|C\|=\|x\|_{1}^{2}
$$

with equality if and only if $M C=C$. Moreover, from the proof of the previous lemma, we conclude that for all $j$ such that the $j$ th column of $M$ is positive, the $j$ th column of $C$ must be zero, which also means that $x_{j}=0$. Thus $\|M x\|_{1}=\|x\|_{1}$ implies that $M x=x$. 
We wish to employ the Banach fixed point theorem to the mapping $\Psi$ on the set $\mathcal{D}$, with the metric that is induced on $\mathcal{D}$ by the following norm

$$
\|B\|=\left\|\left(B_{1}, \ldots, B_{m}\right)\right\|=\max _{1 \leq i \leq m}\left\|B_{i}\right\|
$$

on $\left(\mathcal{R}^{n \times n}\right)^{m}$. Given this, we are now ready to establish that $\Psi^{2}$ is a contraction on the metric space $\mathcal{D}$.

Proposition 5: Let $\Psi$ be the mapping given by (23) and we assume that the transition matrix $P$ is positive. Then there exists a constant $\eta<1$ such that

$$
\left\|\Psi^{2}(D)-\Psi^{2}(E)\right\| \leq \eta\|D-E\|
$$

holds for all $D, E \in \mathcal{D}$.

Proof: We will establish that for every non-zero $B \in \mathcal{B}^{m}$ we have $\left\|\Psi^{2}(B)\right\|<\|B\|$, which implies that there exists a $0<\eta<1$ such that

$$
\left\|\Psi^{2}(B)\right\| \leq \eta\|B\|
$$

since $\mathcal{B}$ is a normed linear space. Clearly, (36) follows from (37), since $D-E \in \mathcal{B}^{m}$. We thus consider any $B=\left(B_{1}, \ldots, B_{m}\right) \neq 0$ such that $B_{i} \in \mathcal{B}$ and compute

$$
\begin{aligned}
\|\Psi(B)\| & =\max _{j}\left\|\sum_{i=1}^{m} \tilde{P}_{i j} M_{i} B_{i} M_{i}^{T}\right\| \\
& \leq \max _{j} \sum_{i=1}^{m} \tilde{P}_{i j}\left\|M_{i} B_{i} M_{i}^{T}\right\| \\
& \leq \max _{j} \sum_{i=1}^{m} \tilde{P}_{i j}\left\|B_{i}\right\| \\
& \leq \max _{j} \sum_{i=1}^{m} \tilde{P}_{i j}\|B\|=\|B\|
\end{aligned}
$$

We will next check under which conditions the equality $\left\|\Psi^{2}(B)\right\|=\|B\|$ can hold. We thus assume that $B \neq 0$ is such that $\|\Psi(\Psi(B))\|=\|B\|$. It follows from

$$
\|B\|=\|\Psi(\Phi(B))\| \leq\|\Psi(B)\| \leq\|B\|
$$

that $\|\Psi(B)\|=\|B\|$. Thus it follows in this situation that all the inequalities in (38)-(41) are actually equalities.

In view of (40) and (41), for some $j$

$$
\sum_{i=1}^{m} \tilde{P}_{i j}\left\|B_{i}\right\|=\max _{i}\left(\left\|B_{i}\right\|\right)=\|B\|
$$

Since we assume that all the $\tilde{P}_{i j}$ are positive, (42) implies

$$
\left\|B_{i}\right\|=\|B\| \quad \text { for every } 1 \leq i \leq m
$$

It then follows from (39) that

$$
\max _{j} \sum_{i=1}^{m} \tilde{P}_{i j}\left\|M_{i} B_{i} M_{i}^{T}\right\|=\max _{j} \sum_{i=1}^{m} \tilde{P}_{i j}\left\|B_{i}\right\|=\|B\|
$$

which together with the positivity of all the $\tilde{P}_{i j}$ imply that

$$
\left\|M_{i} B_{i} M_{i}^{T}\right\|=\|B\|
$$

for all $i$. In view of lemma 3 it therefore follows from (43) and (44) that

$$
M_{i} B_{i} M_{i}^{T}=B_{i} M_{i}^{T}=\left(M_{i} B_{i}^{T}\right)^{T}=\left(M_{i} B_{i}\right)^{T}=B_{i}
$$

namely the equalities

$$
M_{i} B_{i}=B_{i} \quad \text { and } \quad M_{i} B_{i} M_{i}^{T}=B_{i}
$$

hold for all $i$. It follows from (38), (44) and (45) that there exist some $j$ such that

$$
\left\|\sum_{i=1}^{m} P_{i j} B_{i}\right\|=\sum_{i=1}^{m} P_{i j}\left\|B_{i}\right\|=\|B\|
$$

However, this can happen if and only if the following property holds.

$A$ sign condition: For every $r, s \in\{1,2, \ldots, n\}$, there does not exist $1 \leq i, j \leq m$ such that the $(r s)$ th coordinates $\left(B_{i}\right)_{r s}$ and $\left(B_{j}\right)_{r s}$ have opposite signs.

Employing the above argument, and the conclusion (45), to the equality $\|\Psi(\Psi(B))\|=\|\Psi(B)\|$ it follows that for all $k \in\{1,2, \ldots, m\}$

$$
M_{k}\left(\sum_{i=1}^{m} P_{i k} B_{i}\right)=\sum_{i=1}^{m} P_{i k} B_{i}
$$

Now let $l \in\{1,2, \ldots, n\}$ be arbitrary. Then by assumption 2 of our model, there exists some matrix $M_{k} \in \mathcal{M}$ with positive $l$ th column. From (46) we can conclude that the columns of $\sum_{i=1}^{m} P_{i k} B_{i}$ are eigenvectors of the matrix $M_{k}$, which correspond to the eigenvalue 1 . Moreover, they are a convex combination of vectors from $\mathcal{S}$; hence they also belong to $\mathcal{S}$. Using lemma 2 , we can therefore conclude that the $l$ th column of the matrix $\sum_{i=1}^{m} P_{i k} B_{i}$ must vanish, and by employing the above sign condition, it follows that corresponding entries of the various $l$ th columns of the matrices $M_{i}$ do not have opposite signs. This implies that all the entries in the $l$ th columns of the matrices $B_{1}, \ldots, B_{m}$ must vanish. Since $l$ is arbitrary, we conclude that

$$
B_{1}=B_{2}=\cdots=B_{m}=0
$$

This contradiction concludes the proof of the lemma.

Theorem 3: The spectral radius of the restriction of the mapping $\Psi$ to $\mathcal{B}^{m}$ is smaller than 1 . In particular, there exists a unique solution $D^{*}$ for (27), and the iteration scheme

$$
D^{(k+1)}=\Psi\left(D^{(k)}\right), \quad k=0,1,2, \ldots
$$

with the starting point $D^{(0)}=D_{0}$ satisfies

$$
\lim _{k \rightarrow \infty} D^{(k)}=D^{*}
$$

for every $D_{0} \in \mathcal{D}$.

Proof: The proof of this theorem follows the same lines and uses the same arguments as those employed in the proof of theorem 1 .

Proof of proposition 4: Similarly to the proof of proposition 1, let $D_{i}(k)=E\left[W(k) W(k)^{T} \mid A(k)=M_{i}\right]$ and $D(k)=\left(D_{1}(k), \quad \ldots, \quad D_{m}(k) \in\left(\mathbb{R}^{n \times n}\right)^{m}\right.$. Then $D_{i}(k) u=$ $E\left[W(k) W(k)^{T} u \mid A(k)=M_{i}\right]=E\left[W(k) \mid A(k)=M_{i}\right]=V_{i}(k)$ $\rightarrow V_{i}$ as $k \rightarrow \infty$, which means that

$$
\lim _{k \rightarrow \infty} \operatorname{dist}(D(k), \mathcal{D})=0
$$

Let $\varepsilon>0$ and let $k_{0} \in N$ be such that $\left\|D\left(k_{0}\right)-\tilde{D}\right\|<\varepsilon$ for some $\tilde{D} \in \mathcal{D}$. Since the mapping $\Psi$ is non-expansive in the 
norm given by (35) on all $\left(\mathbb{R}^{n \times n}\right)^{m}$ we have

$$
\begin{aligned}
\left\|D\left(k_{0}+r\right)-\Psi^{r}(\tilde{D})\right\| & =\left\|\Psi^{r}\left(D\left(k_{0}\right)-\tilde{D}\right)\right\| \\
& \leq\left\|D\left(k_{0}\right)-\tilde{D}\right\| \leq \varepsilon
\end{aligned}
$$

On the other hand, since $\tilde{D}$ is in $\mathcal{D}$,

$$
\lim _{r \rightarrow \infty} \Psi^{r}(\tilde{D})=\mathcal{D}^{\prime}
$$

exists by the previous theorem. This means that there is $k_{1}$ such that for all $r>k_{1}$,

$$
\left\|\Psi^{r}(\tilde{D})-D^{\prime}\right\| \leq \varepsilon
$$

Now we conclude that for all $r>k_{0}+k_{1}$ :

$$
\begin{aligned}
\left\|D(r)-D^{\prime}\right\| & \leq\left\|D(r)-\Psi^{r-k_{0}}(\tilde{D})\right\|+\left\|\Psi^{r-k_{0}}(\tilde{D})-D^{\prime}\right\| \\
& \leq 2 \varepsilon
\end{aligned}
$$

The last relation means that the sequence $\{D(r)\}$ is a Cauchy, and therefore $\lim _{r \rightarrow \infty} D(r)$ exists and (26) follows. Having this, (27) follows from the continuity of the linear mapping $\Psi$.

Theorem 4: Under assumption 2 and the positivity of the transition matrix $P$, the asymptotic behaviour of the variance

$$
\operatorname{Var}(W(N))=E\left[W(N) W(N)^{T}\right]-E[W(N)] E[W(N)]^{T}
$$

is given by

$$
\lim _{N \rightarrow \infty} \operatorname{Var}(W(N))=\sum_{i=1}^{m} \rho_{i} D_{i}^{*}-\left(\sum_{i=1}^{m} \rho_{i} V_{i}^{*}\right)\left(\sum_{i=1}^{m} \rho_{i} V_{i}^{*}\right)^{T}
$$

where $D^{*}=\left(D_{1}^{*}, \ldots, D_{m}^{*}\right) \in \mathcal{D}$ is the unique solution of (27), and $\rho=\left(\rho_{1}, \ldots, \rho_{m}\right)$ is the Perron eigenvector of the transition matrix $\left(P_{i j}\right)$.

Proof: We have the following equalities:

$$
\begin{aligned}
& \lim _{N \rightarrow \infty} \operatorname{Var}(W(N)) \\
& =\lim _{N \rightarrow \infty} E\left[W(N) W(N)^{T}\right]-\lim _{N \rightarrow \infty} E[W(N)] E[W(N)]^{T} \\
& =\lim _{N \rightarrow \infty} \sum_{i=1}^{m} E\left[W(N) W(N)^{T} \mid A(N)=M_{i}\right] \\
& \quad \times P\left[A(N)=M_{i}\right]-\lim _{N \rightarrow \infty} E[W(N)] E[W(N)]^{T} \\
& =\sum_{i=1}^{m} \rho_{i} D_{i}^{*}-\left(\sum_{i=1}^{m} \rho_{i} V_{i}^{*}\right)\left(\sum_{i=1}^{m} \rho_{i} V_{i}^{*}\right)^{T}
\end{aligned}
$$

Corollary 3: Let assumption 1 hold, so that the probability that $A(k)=M_{i}$ is equal to $\rho_{i}$ for every $k \geq 0$ and $1 \leq i \leq m$. Then the asymptotic behaviour of $\operatorname{Var}(W(k))$ is given by

$$
\lim _{N \rightarrow \infty} \operatorname{Var}(W(N))=\bar{D}-\bar{V} \bar{V}^{T}
$$

where $\bar{V}$ is the unique stochastic eigenvector of the matrix $\sum_{i=1}^{m} \rho_{i} M_{i}$, and $\bar{D}$ is the unique solution of the matrix equation

$$
\sum_{i=1}^{m} \rho_{i} M_{i} D M_{i}^{T}=D
$$

which satisfies $D u=\bar{V}$. Moreover, $\bar{D}$ is the unique eigenvector corresponding to eigenvalue 1 and satisfying $D u=\bar{V}$ of the linear mapping

$$
D \mapsto \sum_{i=1}^{m} \rho_{i} M_{i} D M_{i}^{T}
$$

defined on $\mathbb{R}^{n \times n}$.

Proof: The sequence $\{A(k)\}$ of i.i.d. random matrices can be seen as Markov chain on the set $\mathcal{M}=\left\{M_{i}: \rho_{i}>0\right\}$ with the $m \times m$ transition matrix $P$ given by $P_{i j}=\rho_{j}$. Since $P$ is positive for every $i$ and $j$, it follows that $\tilde{P}_{i j}=\rho_{i} P_{i j} / \rho_{j}=\rho_{i}>0$. We look for a solution of (27) for which all the components $D_{i}$ are the same, say equal to $\bar{D}$. This yields the equation

$$
\bar{D}=\sum_{i=1}^{m} \rho_{i} M_{i} \bar{D} M_{i}^{T}
$$

which implies the first assertion of the corollary.

For the second assertion we represent the linear mapping in (49) by an $n^{2} \times n^{2}$ matrix with non-negative entries, call it $T$, and apply Perron-Frobenius theorem to $T$. By theorem 3 the spectral radius of the restriction of $T$ to $\mathcal{B}$ is smaller than 1 , but by $(50)$ we have that $\bar{D}$ is an eigenvector corresponding to the eigenvalue 1 . Since the iterations of the mapping (49) converge to $\bar{D}$, it follows that 1 is the unique eigenvector satisfying $\bar{D} u=\bar{V}$. The second assertion follows.

Example 1: In this example we illustrate how the previous results can be applied. We consider a network where the bottleneck router operates according to assumption 2 . In particular, consider a network of five flows with additive increase parameters $\alpha=[5,4,3,2,1]$, multiplicative decrease parameters given by $\beta=[1 / 3,2 / 4,3 / 5,4 / 6$, $5 / 7]$, and with corresponding vector $\gamma$ given by $\gamma=[1 / 60$, $1 / 70,1 / 80,1 / 90,1 / 100]$. We assume that at congestion events the router drops packets from only one flow. Thus the set $\mathcal{M}$ has five elements:

$$
\begin{aligned}
M_{1}= & {\left[\begin{array}{ccccc}
0.5054 & 0 & 0 & 0 & 0 \\
0.1475 & 1.0000 & 0 & 0 & 0 \\
0.1291 & 0 & 1.0000 & 0 & 0 \\
0.1147 & 0 & 0 & 1.0000 & 0 \\
0.1033 & 0 & 0 & 0 & 1.0000
\end{array}\right] } \\
M_{2}= & {\left[\begin{array}{ccccc}
1.0000 & 0.1291 & 0 & 0 & 0 \\
0 & 0.6106 & 0 & 0 & 0 \\
0 & 0.0968 & 1.0000 & 0 & 0 \\
0 & 0.0860 & 0 & 1.0000 & 0 \\
0 & 0.0774 & 0 & 0 & 1.0000
\end{array}\right] } \\
M_{3}= & {\left[\begin{array}{ccccc}
1.0000 & 0 & 0.1033 & 0 & 0 \\
0 & 1.0000 & 0.0885 & 0 & 0 \\
0 & 0 & 0.6774 & 0 & 0 \\
0 & 0 & 0.0688 & 1.0000 & 0 \\
0 & 0 & 0.0620 & 0 & 1.0000
\end{array}\right] }
\end{aligned}
$$




$$
\begin{aligned}
M_{4} & =\left[\begin{array}{ccccc}
1.0000 & 0 & 0 & 0.0860 & 0 \\
0 & 1.0000 & 0 & 0.0738 & 0 \\
0 & 0 & 1.0000 & 0.0645 & 0 \\
0 & 0 & 0 & 0.7240 & 0 \\
0 & 0 & 0 & 0.0516 & 1.0000
\end{array}\right] \\
M_{5} & =\left[\begin{array}{ccccc}
1.0000 & 0 & 0 & 0 & 0.0738 \\
0 & 1.0000 & 0 & 0 & 0.0632 \\
0 & 0 & 1.0000 & 0 & 0.0553 \\
0 & 0 & 0 & 1.0000 & 0.0492 \\
0 & 0 & 0 & 0 & 0.7585
\end{array}\right]
\end{aligned}
$$

Let the transition matrix $P$ be given by

$$
P=\left[\begin{array}{lllll}
0.2667 & 0.2467 & 0.2133 & 0.1667 & 0.1067 \\
0.2606 & 0.2424 & 0.2121 & 0.1697 & 0.1152 \\
0.2526 & 0.2368 & 0.2105 & 0.1737 & 0.1263 \\
0.2444 & 0.2311 & 0.2089 & 0.1778 & 0.1378 \\
0.2370 & 0.2259 & 0.2074 & 0.1815 & 0.1481
\end{array}\right]
$$

Then

$$
\lim _{k \rightarrow \infty} E(W(k))=V^{*}=\left[\begin{array}{l}
0.2359 \\
0.2295 \\
0.2124 \\
0.1852 \\
0.1370
\end{array}\right]
$$

The meaning of this result is that the first flow should expect to get $23.59 \%$ of bandwidth, while the fifth flow should expect to get $13.70 \%$ of the bandwidth over the bottleneck link, provided that they last long enough. The asymptotic behaviour of the variance of $W(k)$ in this example is given by

$$
\begin{aligned}
& \lim _{k \rightarrow \infty} \operatorname{Var}(W(k)) \\
& =\left[\begin{array}{rrrrr}
0.0144 & -0.0061 & -0.0042 & -0.0027 & -0.0013 \\
-0.0061 & 0.0118 & -0.0029 & -0.0019 & -0.0009 \\
-0.0042 & -0.0029 & 0.0089 & -0.0012 & -0.0005 \\
-0.0027 & -0.0019 & -0.0012 & 0.0060 & -0.0002 \\
-0.0013 & -0.0009 & -0.0005 & -0.0002 & 0.0030
\end{array}\right]
\end{aligned}
$$

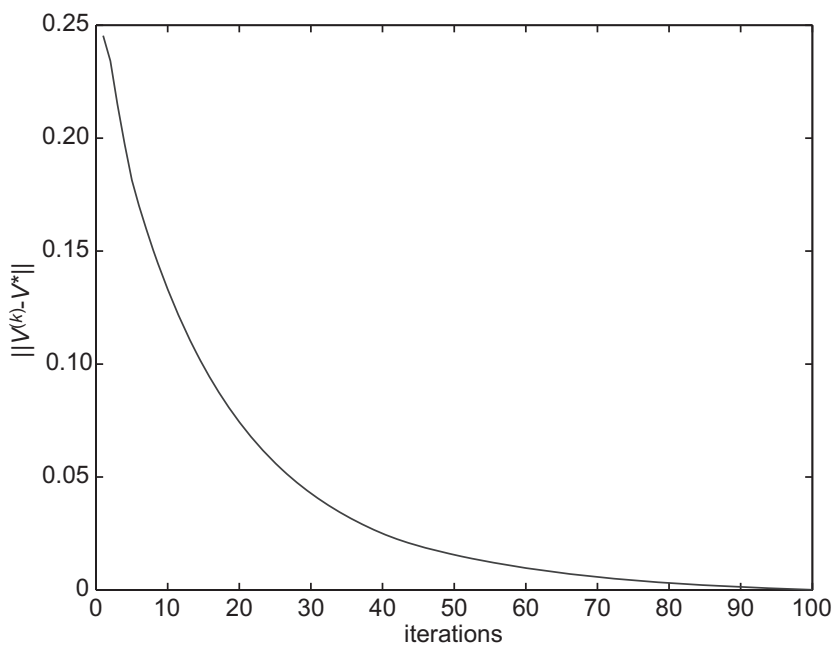

Fig. 2 Evolution of $\left\|V^{(k)}-V^{*}\right\|_{I}$

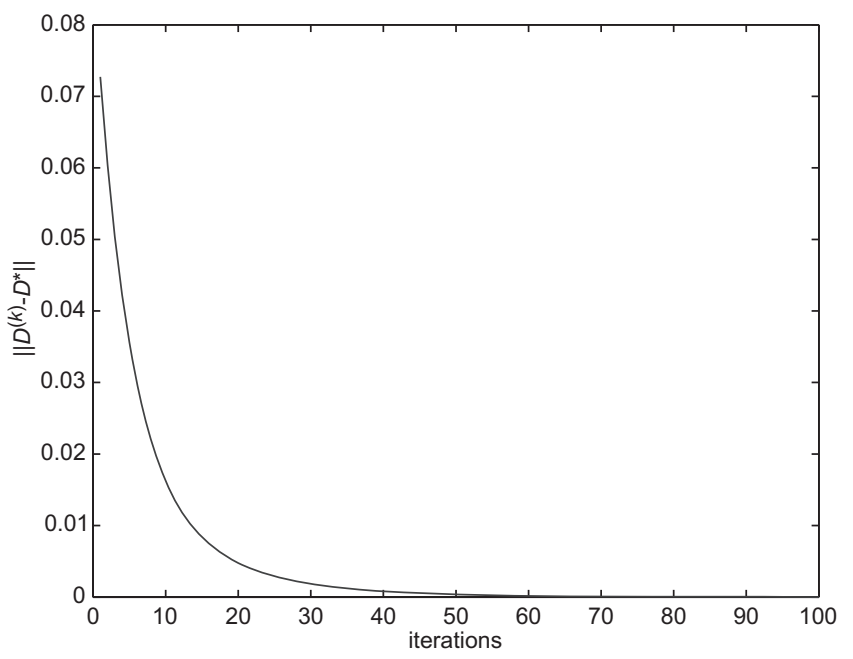

Fig. 3 Evolution of $\left\|D^{(k)}-D^{*}\right\|$

The rate at which $V^{(k)}$ and $D^{(k)}$ converge to their equilibrium values is depicted graphically in Figs. 2 and 3, respectively.

\section{A useful extension}

In this Section we will extend the results of the previous sections in the following sense. We will consider a transition probability matrix $P$ that does not necessarily have positive entries, but is rather primitive, namely $P^{s}>0$, for some integer $s \geq 1$. We note here that if $P$ is primitive then $\tilde{P}$ is primitive too, since they have the same zeronon-zero pattern.

Lemma 4: If $P$ is a primitive matrix such that $P^{s}>0$ for some positive integer $s$, then $\Phi^{2 s}$ is a contraction on $\Sigma^{m}$.

Proof: We first note that for all $k, j, l \in\{1,2, \ldots, m\}$, there is sequence $(i)=\left(i_{1}, i_{2}, \ldots, i_{2 s-1}\right)$ of indices that contain $l$, such that

$$
\tilde{P}_{k i_{2 s-1}} \tilde{P}_{i_{2 s-1} i_{2 s-2}} \cdots \tilde{P}_{i_{2} i_{1}} \tilde{P}_{i_{1} j}>0
$$

Indeed, since $P^{s}>0$ there must exist sequences $\left(i^{\prime}\right)=\left(i_{1}^{\prime}, \ldots, i_{s-1}^{\prime}\right)$ and $\left(i^{\prime \prime}\right)=\left(i_{1}^{\prime \prime}, \ldots, i_{s-1}^{\prime \prime}\right)$ such that

$$
\tilde{P}_{k i_{s-1}^{\prime}} \tilde{P}_{i_{s-1}^{\prime} i_{s-2}^{\prime}} \cdots \tilde{P}_{i_{2}^{\prime} i_{1}^{\prime}} \tilde{P}_{i_{1}^{\prime} l}>0
$$

and

$$
\tilde{P}_{l_{s-1}^{\prime \prime}} \tilde{P}_{i_{s-1}^{\prime \prime} i_{s-2}^{\prime \prime}} \cdots \tilde{P}_{i_{2}^{\prime \prime} i_{1}^{\prime \prime}} \tilde{P}_{i_{1}^{\prime \prime} j}>0
$$

implying the existence of a sequence $(i)$ with the desired property.

As we noted in Section 2, proving that $\Phi^{2 s}$ is a contraction on the metric space $\Sigma^{m}$ is equivalent to proving that it is a contraction on the vector space $\mathcal{S}^{m}$, and here we establish the latter. Thus for an arbitrary $W \in \mathcal{S}^{m}$ the $j$ component of $\Phi^{2 s}(W)$ has the form

$$
\left(\Phi^{2 s}(W)\right)_{j}=\sum_{(i)_{2 s}} \tilde{P}_{i_{2 s} i_{2 s-1}} \tilde{P}_{i_{2 s-1} i_{2 s-2}} \cdots \tilde{P}_{i_{1} j} M_{i_{1}} M_{i_{2}} \cdots M_{i_{2 s}} W_{i_{2 s}}
$$

where we denote by $(i)_{2 s}$ a sequence of indices that has length $2 s$, and the summation is over all possible sequences $(i)_{2 s}$. The sum in the last equality can be 
rewritten as

$$
\left(\Phi^{2 s}(W)\right)_{j}=\sum_{k=1}^{m} \sum_{(i)} \tilde{P}_{k i_{2 s-1}} \cdots \tilde{P}_{i_{1} j} M_{i_{1}} \cdots M_{i_{2 s-1}} M_{k} W_{k}
$$

The inner sum in (51) is over all the sequences of indices (i) that have the length $2 s-1$. Having this in mind, we write

$$
\begin{aligned}
& \left\|\Phi^{2 s}(W)\right\| \\
& \quad=\max _{j}\left\|\sum_{k=1}^{m} \sum_{(i)} \tilde{P}_{k i_{2 s-1}} \cdots \tilde{P}_{i_{1} j} M_{i_{1}} \cdots M_{i_{2 s-1}} M_{k} W_{k}\right\|_{1} \\
& \leq \max _{j} \sum_{k=1}^{m} \sum_{(i)} \tilde{P}_{k i_{2 s-1}} \ldots \tilde{P}_{i_{1} j}\left\|M_{i_{1}} \cdots M_{i_{2 s-1}} M_{k} W_{k}\right\|_{1} \\
& \quad \leq \max _{j} \sum_{k=1}^{m} \sum_{(i)} \tilde{P}_{k i_{2 s-1}} \tilde{P}_{i_{2 s-1} i_{2 s-2}} \cdots \tilde{P}_{i_{1} j}\left\|W_{k}\right\|_{1} \\
& \quad \leq \max _{j} \sum_{k=1}^{m} \sum_{(i)} \tilde{P}_{k i_{2 s-1}} \tilde{P}_{i_{2 s-1} i_{2 s-2}} \cdots \tilde{P}_{i_{1} j}\|W\|=\|W\|
\end{aligned}
$$

We will next establish that $\left\|\Phi^{2 s}(W)\right\|=\|W\|$ implies that $W=0$, which will conclude the proof of the lemma. If $W \in \mathcal{S}^{m}$ satisfies $\left\|\Phi^{2 s}(W)\right\|=\|W\|$ then all the previous inequalities (52)-(54) are actually equalities. This means that there exists some $j \in\{1,2, \ldots, m\}$ for which all the above maxima are attained at this $j$. For a $k \in\{1,2, \ldots$, $m\}$ and a sequence $(i)$ of indices we denote

$$
Q_{k j}((i))=\tilde{P}_{k i_{2 s-1}} \tilde{P}_{i_{2 s-1} i_{2 s-2}} \cdots \tilde{P}_{i_{1} j}
$$

It follows from $P^{2 s}=\left(P^{s}\right)^{2}>0$ that for each $k \in\{1,2, \ldots$, $m\}$ we have $\sum_{(i)} Q_{k j}((i))>0$. From this property together with

$$
\sum_{k=1}^{m}\left\|W_{k}\right\|_{1}\left(\sum_{(i)} Q_{k j}((i))\right)=\max _{k}\left\|W_{k}\right\|_{1}=\|W\|
$$

and

$$
\sum_{k=1}^{m} \sum_{(i)} Q_{k j}((i))=1
$$

we can conclude that for every $k \in\{1,2, \ldots, m\}$

$$
\left\|W_{k}\right\|_{1}=\|W\|
$$

It follows from the equality

$$
\begin{aligned}
& \sum_{k=1}^{m} \sum_{(i)} Q_{k j}((i))\left\|M_{i_{1}} M_{i_{2}} \cdots M_{i_{2 s-1}} M_{k} W_{k}\right\|_{1} \\
& \quad=\sum_{k=1}^{m} \sum_{(i)} Q_{k j}((i))\left\|W_{k}\right\|_{1}
\end{aligned}
$$

that for every sequence $(i)$ such that $Q_{k j}((i))>0$ the following holds

$$
\left\|M_{i_{1}} M_{i_{2}} \cdots M_{i_{2 s-1}} M_{k} W_{k}\right\|_{1}=\left\|W_{k}\right\|_{1}
$$

which in turn implies

$$
\begin{gathered}
\left\|M_{i_{1}} \cdots M_{i_{2 s-1}} M_{k} W_{k}\right\|_{1}=\left\|M_{i_{2}} \cdots M_{i_{2 s-1}} M_{k} W_{k}\right\|_{1} \\
\cdots=\left\|M_{i_{2 s-1}} M_{k} W_{k}\right\|_{1}=\left\|M_{k} W_{k}\right\|_{1}=\left\|W_{k}\right\|
\end{gathered}
$$

Employing lemma 1, we conclude that for all sequences $(i)$ with $Q_{k j}((i))>0$ :

$$
W_{k}=M_{k} W_{k}=M_{i_{2 s-1}} W_{k}=\cdots=M_{i_{1}} W_{k}
$$

Recall now that for arbitrary $k$ and $l$ there exists a sequence (i) which contains $l$ such that $Q_{k j}((i))>0$. Using (58) we conclude that

$$
M_{l} W_{k}=W_{k}, \quad \forall k, l \in\{1,2, \ldots, m\}
$$

and the relations (59) imply

$$
W_{1}=\cdots=W_{m}=0
$$

Indeed, for each $h \in\{1,2, \ldots, m\}$ there exists a matrix $M_{l} \in \mathcal{M}$ with positive $h$ column (by assumption 2). Thus, lemma 2 implies that the $h$ coordinate of each $W_{k}$ vanishes, and since $h$ is arbitrary, (60) follows. The proof of the lemma is complete.

The following result may be established by using the same arguments as those employed in proving the previous lemma, and we will not repeat it here.

Lemma 5: If $P$ is a primitive matrix, such that $P^{s}>0$ for some positive integer $s$, then $\Psi^{2 s}$ is a contraction on $\mathcal{D}$.

Inspecting the proof of theorem 1, we realise that we did not use any special properties of the second power in deriving the proof while using the contractive property of $\Phi^{2}$. Namely, for any positive integer $q$, if $\Phi^{q}$ is contractive on $\Sigma^{m}$, then theorem 1 follows. Similarly, if $\Psi^{q}$ is contractive on $\mathcal{D}$ for some positive integer $q$ then theorem 3 follows. As a consequence of the previous two lemmas, we have the following results.

Theorem 5: Let assumption 2 hold and suppose that the transition matrix $P$ is primitive, so that there exists an integer $s \geq 1$ such that $P^{s}$ has positive entries. Then the spectral radius of the restriction of $\Phi$ to $\mathcal{S}^{m}$ is smaller than 1. In particular there exists a unique solution $V^{*}$ for (7), and the iteration scheme

$$
V^{(k+1)}=\Phi\left(V^{(k)}\right), \quad k=0,1,2, \ldots
$$

with any starting point $V^{(0)}=V_{0}$ in $\Sigma$

$$
\lim _{k \rightarrow \infty} V^{(k)}=V^{*}
$$

Moreover, the asymptotic behaviour of the expectation of the random variable $W(N)$ is given by

$$
\lim _{N \rightarrow \infty} E(W(N))=\sum_{i=1}^{m} \rho_{i} V_{i}^{*}
$$

where $V^{*}=\left(V_{1}^{*}, \ldots, V_{m}^{*}\right) \in \Sigma^{m}$ is the unique solution of (7), and $\rho=\left(\rho_{1}, \ldots, \rho_{m}\right)$ is the Perron eigenvector of the transition probability matrix $\left(P_{i j}\right)$.

Theorem 6: Let assumption 2 hold, and assume that the transition probability matrix $P$ is primitive. Then the spectral radius of the restriction of the mapping $\Psi$ to $\mathcal{B}^{m}$ is smaller than 1 . In particular there exists a unique solution $D^{*}$ for (27), and the iteration scheme

$$
D^{(k+1)}=\Psi\left(D^{(k)}\right), \quad k=0,1,2, \ldots
$$

with the starting point $D^{(0)}=D_{0}$ satisfies

$$
\lim _{k \rightarrow \infty} D^{(k)}=D^{*}
$$


for every $D_{0} \in \mathcal{D}$. Moreover, the asymptotic behaviour of the variance

$$
\operatorname{Var}(W(N))=E\left[W(N) W(N)^{T}\right]-E[W(N)] E[W(N)]^{T}
$$

is given by

$$
\lim _{N \rightarrow \infty} \operatorname{Var}(W(N))=\sum_{i=1}^{m} \rho_{i} D_{i}^{*}-\left(\sum_{i=1}^{m} \rho_{i} V_{i}^{*}\right)\left(\sum_{i=1}^{m} \rho_{i} V_{i}^{*}\right)^{T}
$$

where $D^{*}=\left(D_{1}^{*}, \ldots, D_{m}^{*}\right) \in \mathcal{D}$ is the unique solution of (27), and $\rho=\left(\rho_{1}, \ldots, \rho_{m}\right)$ is the Perron eigenvector of the transition matrix $\left(P_{i j}\right)$.

\section{$5 \quad R$-model}

In the previous sections we considered the process $\{(A(k)$, $W(k))\}_{k=0}^{\infty}$ under the assumption that $\{A(k)\}_{k=0}^{\infty}$ is a Markov process, and the distribution of $W(k+1)$ is determined by the value of $A(k)$ and the distribution of $W(k)$. In this model, the emphasis is put on the process $\{A(k)\}_{k=0}^{\infty}$, and $\{W(k)\}_{k=0}^{\infty}$ may be considered as a 'shadow' of it since the properties of $\{W(k)\}_{k=0}^{\infty}$ are derived from the distribution of $\{A(k)\}_{k=0}^{\infty}$.

However, one can construct a router such that the probability that a packet will be dropped at the $k$ th congestion event depends on the information provided by the vector $W(k)$, whose $j$ th coordinate is equal to the throughput of the $j$ th flow at the $k$ th congestion. We thus assume throughout this section that assumption 3 holds, and we will describe it again below.

When we consider the model under assumption 3, which we call the $R$-model, we assume that the value of $W(k)$ at the $k$ th congestion event, say $W(k)=w$, determines the distribution of $A(k)$. Namely, there exist continuous functions $w \mapsto p_{i}(w) \in \mathbb{R}^{+}$on $\Sigma$ such that

$P\left[A(k)=M_{i} \mid W(k)=w\right]=p_{i}(w) \quad(\forall 1 \leq i \leq m) \quad(\forall w \in \Sigma)$

and

$$
\sum_{i=1}^{m} p_{i}(w)=1 \quad \text { for every } w \in \Sigma
$$

In order to ensure that each flow has non-zero probability to detect a drop we assume that for each flow $i$ there exist matrix in $\mathcal{M}$ with positive $i$ th column.

We begin this section by proving that for any initial distribution of $W(0)$ almost all products $\{A(k) \cdots A(0)\}_{k \in N}$ are weakly ergodic. Recall that a sequence $\left\{Q_{k}\right\}_{k \in N}$ of column-stochastic matrices is called weakly ergodic if

$$
\lim _{k \rightarrow \infty} \operatorname{dist}\left(Q_{k}, \mathcal{R}\right)=0
$$

where we denote by $\mathcal{R}$ set of rank-1 column stochastic matrices. For any column stochastic matrix $Q$, we know that $Q(\mathcal{S}) \subset \mathcal{S}$ (see the proof of proposition 2). Thus the restriction of $Q$ to $\mathcal{S}$ is a mapping to itself, and we denote this map by $\tilde{Q}$. It follows from the definition of weak ergodicity given above that the sequence $\left\{Q_{k}\right\}_{k \in N}$ is weakly ergodic if and only if

$$
\lim _{k \rightarrow \infty} \tilde{Q}_{k}=0
$$

(see [8]).
Recall also that a linear operator on a vector space $V$ is called paracontractive with respect to norm $\|\cdot\|$ if for all $x \in V$

$$
V x \neq x \Longrightarrow\|V x\|<\|x\|
$$

The main tool in establishing almost sure weak ergodicity will be the following result, which is given in [6].

Theorem 7: Let $\|\cdot\|$ be a norm on $\mathbb{R}^{m}$ and let $\mathcal{F} \subset \mathbb{R}^{m \times m}$ be a finite set of linear operators that are paracontractive with respect to $\|\cdot\|$. Then for any sequence $\left\{A_{k}\right\}_{k \in N} \subset \mathcal{F}^{N}$, the sequence of left products $\left\{A_{k} A_{k-1} \cdots A_{1}\right\}_{k \in N}$ converges.

Proposition 6: Let the random variable $W(0)$ have arbitrary distribution on $\Sigma$. Under assumption 3 , the sequence of products $\{A(k) A(k-1) \cdots A(0)\}_{k \in N}$ is weakly ergodic with probability 1 , that is,

$$
\lim _{k \rightarrow \infty} \tilde{A}(k) \tilde{A}(k-1) \cdots \tilde{A}(0)=0 \quad \text { almost surely }
$$

Proof: From the assumption of positivity and the continuity of the mappings $p_{i}$ on the compact set $\Sigma$ it follows that

$$
\eta=\inf \left\{p_{i}(s) \mid s \in \Sigma, i \in\{1, \ldots, m\}\right\}>0
$$

This means that $p\left(A(k) \neq M_{i}\right) \leq 1-\eta<1$ for every $1 \leq i \leq m$. For every such $i$ let $T_{i}$ be a matrix with positive $i$ th column. Then with probability 1 the matrix $T_{i}$ appears infinitely often in $\{A(k)\}_{k=0}^{\infty}$. We will next establish weak ergodicity for left products $\left\{A_{k} \ldots A_{1} A_{0}\right\}_{k \in N}$ whenever for every $i$ there exist matrix $T_{i}$ with positive $i$ th column that occurs infinitely often in sequence $A_{0}, A_{1}, A_{2}, \ldots$

By lemma 1 , for all $M \in \mathcal{M}, \tilde{M}$ is paracontracting on $\mathcal{S}$ with respect to the $L_{1}$ norm. By theorem 7 it follows that the sequence $\left\{\tilde{A}_{k} \tilde{A}_{k-1} \cdots \tilde{A}_{0}\right\}_{k \in N}$ is convergent, and we claim that the limit is zero. To show this let $s \in \mathcal{S}$. Then there exist a $y \in \mathcal{S}$ such that $y=\lim _{k \rightarrow \infty} \tilde{A}_{k} \tilde{A}_{k-1} \cdots \tilde{A}_{0} s$. For any fixed $i$ let $\left\{A_{n_{k}}\right\}_{k \in N}$ be a subsequence of $\left\{A_{k}\right\}_{k \in N}$ with $A_{n_{k}}=T_{i}$. Then

$$
y=\lim _{k \rightarrow \infty} \tilde{A}_{n_{k}} \tilde{A}_{n_{k}-1} \cdots \tilde{A}_{0} s=T_{i} \lim _{k \rightarrow \infty} \tilde{A}_{n_{k}-1} \cdots \tilde{A}_{0} s=T_{i} y
$$

hence $T_{i} y=y$. But by lemma 2 , the $i$ th coordinate of $y$ must be zero. Since $i$ is arbitrary, it follows that $y=0$. We have thus established that $\lim _{k \rightarrow \infty} \tilde{A}(k) \tilde{A}(k-1) \cdots \tilde{A}(0)=0$, which implies the assertion of the proposition.

Comment: The previous proposition is also established in [23], under assumption 1, that is, when all the functions $p_{i}$ are constant.

Note that, in a sense, under assumption 3 , the roles of $\{A(k)\}_{k=0}^{\infty}$ and $\{W(k)\}_{k=0}^{\infty}$ are interchanged compared to their roles in the model under assumption 2: the emphasis is put on $\{W(k)\}_{k=0}^{\infty}$, and $\{A(k)\}_{k=0}^{\infty}$ is considered as its shadow process.

We will henceforth restrict attention to stationary processes. The process $\{A(k), W(k)\}_{k=0}^{\infty}$ is Markovian in the compact state space $\{1,2, \ldots, m\} \times \Sigma$, and we will next establish that it has a unique equilibrium distribution

$$
\left\{\rho_{1}, \ldots, \rho_{m}\right\} \times\left(\lambda_{1}(d w), \ldots, \lambda_{m}(d w)\right)
$$

namely the probability that $A(k)=M_{i}$ and $W(k) \in U$ is equal, in the limit where $k \rightarrow \infty$, to $\rho_{i} \lambda_{i}(U)$. The equilibrium measure is defined on the set of limit points of $\{W(k)\}_{k=0}^{\infty}$, and for a prescribed $W_{0}=s$ we denote by $F(s)$ the set of all limit points of sequences $\{W(k)\}_{k=0}^{\infty}$ with $W(0)=s$. We use the following terminology and say that 
weak ergodicity holds for $\left\{M_{1}, \ldots, M_{m}\right\}=\mathcal{M}$ if every product

$$
A_{k} A_{k-1} \cdots A_{0}, \quad A_{k} \in \mathcal{M} \quad \text { for every } k \geq 0
$$

in which each $M_{i}$ appears infinitely often is weakly ergodic.

Proposition 7: Suppose that $p_{i}(w)>0$ for every $1 \leq i \leq m$ and $w \in \Sigma$, and assume that weak ergodicity holds for $\left\{M_{1}, \ldots, M_{m}\right\}$. Then

$$
F\left(s_{1}\right)=F\left(s_{2}\right)=: F \quad \text { for every } s_{1}, s_{2} \in \Sigma
$$

Thus $F$ is the smallest closed subset of $\Sigma$ that is invariant under each $M_{i} \in \mathcal{M}$, so that it satisfies

$$
F=\bigcup_{i=1}^{m} M_{i}(F)
$$

and it is the support of the unique equilibrium invariant measure $\left(\lambda_{1}(d w), \ldots, \lambda_{m}(d w)\right)$.

Proof: For a prescribed starting point $W_{0}=s$ we define the sequence of subsets $F_{k}(s) \subset \Sigma$ as

$F_{0}(s)=\{s\}, \quad F_{k+1}(s)=\bigcup_{i=1}^{m} M_{i}\left(F_{k}(s)\right), \quad k=0,1,2, \ldots$

Then $F(s)$ may be expressed in the form

$$
F(s)=\bigcap_{p=1}^{\infty}\left(\operatorname{cl} \bigcup_{k=p}^{\infty} F_{k}(s)\right)
$$

Denote by $h(\cdot, \cdot)$ the Hausdorff metric in $\Sigma$. It then follows from the weak ergodicity of $\mathcal{M}$ that

$$
h\left(F_{k}\left(s_{1}\right), F_{k}\left(s_{2}\right)\right) \longrightarrow 0 \quad \text { as } k \longrightarrow \infty
$$

This follows from the fact that each point in $F_{k}(s)$ is of the form

$$
M_{i_{k}} M_{i_{k-1}} \cdots M_{i_{1}} s
$$

for some matrices $M_{i_{j}} \in \mathcal{M}, 1 \leq j \leq k$, and that

$$
M_{i_{k}} M_{i_{k-1}} \cdots M_{i_{1}} s_{1}-M_{i_{k}} M_{i_{k-1}} \cdots M_{i_{1}} s_{2} \longrightarrow 0 \quad \text { as } k \longrightarrow \infty
$$

by weak ergodicity. It follows from (69) that the Hausdorff distance between $\bigcup_{k=p}^{\infty} F_{k}\left(s_{1}\right)$ and $\bigcup_{k=p}^{\infty} F_{k}\left(s_{2}\right)$ is arbitrarily small provided $p$ is sufficiently large. In view of (68) it follows that $F\left(s_{1}\right)=F\left(s_{2}\right)$ for every $s_{1}, s_{2} \in \Sigma$, establishing (66) and concluding the proof of the proposition.

The dynamics of $\left\{(A(k), W(k)\}_{k=0}^{\infty}\right.$ can be described as follows. For a vector $W(k)=w$ at the instant of time $k$, choose a matrix $A(k)$ from $\mathcal{M}$ according to the distribution $\left\{p_{i}(w)\right\}_{i=1}^{m}$, and set $W(k+1)=A(k) w$. We then follow the steps

$$
\begin{aligned}
& W(0) \longrightarrow A(0) \longrightarrow W(1)=A(0) W(0) \longrightarrow A(1) \longrightarrow \cdots \\
& \quad \longrightarrow W(k) \longrightarrow A(k) \longrightarrow W(k+1)=A(k) W(k) \longrightarrow \cdots
\end{aligned}
$$

We restrict attention only to the terms $W(k)$ in the chain of variables (70), and if $W(0) \sim\left(\lambda_{1}, \ldots, \lambda_{m}\right)$ then $\{W(k)\}_{k=0}^{\infty}$ turns out to be a stationary Markov process.

We now view the dynamics in a different manner, and this time we focus on the terms $A(k)$ in the above chain (70). If in the outset we restrict attention to stationary processes, then the distribution of each variable $W(k)$ is $\left(\lambda_{1}(d w), \ldots, \lambda_{m}(d w)\right)$. Assuming this, we restrict attention only to the variables $A(k)$ in (70), which turns out to be a stationary Markov chain in $\mathcal{M}$ provided that we take the initial distribution $A(0) \sim \rho$. We thus suppose that $W(k) \sim\left(\lambda_{1}, \ldots, \lambda_{m}\right)$, and that $A(k)=M_{i}$ for some $1 \leq i \leq m$. This determines the distribution of $W(k+1)=M_{i} W(k)$, as well as the distribution of $A(k+1)$. More explicitly, we define $P_{i j}=E p_{j}\left(M_{i} W(k)\right)$ where $E$ denotes the expectation operation with respect to the distribution $\lambda(d w)$, namely

$$
P_{i j}=\int p_{j}\left(M_{i} w\right) \lambda_{i}(d w)
$$

Although we defined $P_{i j}=E p_{j}\left(M_{i} W(k)\right), P_{i j}$ in (71) does not actually depend on $k$ since all the variables $W(k)$ have the same distribution $\lambda$. However, we have to verify that our construction does yield this distribution to all $W(k)$. But indeed, since $\rho \times\left(\lambda_{1}, \ldots, \lambda_{m}\right)$ is an equilibrium distribution for the Markov process $\{A(k), W(k)\}_{k=0}^{\infty}$, it follows that if we have $A(k) \sim \rho$, then the distribution of $W(k+1)$ is $\left(\lambda_{1}, \ldots, \lambda_{m}\right)$, and that of $A(k+1)$ is $\rho$. We summarise the above discussion in the following.

Theorem 8: The matrix $P$ is a transition probability matrix of a stationary Markov chain $\{A(k)\}_{k=0}^{\infty}$ in $\mathcal{M}$ with stationary distribution $A(k) \sim \rho$. This Markov chain consist of the $A(k)$ terms in the process $\{A(k), W(k)\}_{k=0}^{\infty}$, which describes the $R$-model of the process.

We are interested in the asymptotic behaviour of $W(N)$ where $N \rightarrow \infty$, which in view of $W(k+1)=A(k) W(k)$, reduces to the study of the asymptotic distribution of the products

$$
\left(\prod_{k=0}^{N} A(k)\right) W(0)
$$

when $N \rightarrow \infty$. Since weak ergodicity holds for the matrix products $\prod_{k=0}^{N} A(k)$, it follows that the asymptotic behaviour of the expressions in (72) does not depend on $W(0)$ there, and we consider these expressions with an arbitrary choice of $W(0) \in \Sigma$. Although $\{A(k)\}_{k=0}$ is a stationary process, the process $\{A(N) \cdots A(0) W\}$ is not stationary, and we define

$$
V_{i}^{N}=E\left[A(N-1) A(N-2) \cdots A(0) W(0) \mid A(N)=M_{i}\right]
$$

where the expectation is with respect to the measure in which $\{A(k)\}_{k=0}^{\infty}$ is a stationary Markov chain with the transition probability matrix $P$ in (71). Associated with this $P$ is the matrix $\tilde{P}$ of backward probabilities, so that $\tilde{P}_{i j}$ is the probability of having $A(k)=M_{i}$ given that $A(k+1)=M_{j}$. Thus assuming $A(N+1)=M_{j}$, it follows from (73) that

$$
\begin{aligned}
V_{j}^{N+1}= & E\left[A(N) A(N-1) \cdots A(0) W(0) \mid A(N+1)=M_{j}\right] \\
= & \sum_{i=1}^{m} \tilde{P}_{i j} E\left[M_{i} A(N-1) \cdots A(0) W(0) \mid A(N)=M_{i},\right. \\
& \left.A(N+1)=M_{j}\right] \\
= & \sum_{i=1}^{m} \tilde{P}_{i j} M_{i} E\left[A(N-1) \cdots A(0) W(0) \mid A(N)=M_{i}\right]
\end{aligned}
$$

where in the last equality we have used the Markov property. Equating the first and last terms and using (73), we 
obtain the relations

$$
V_{j}^{N+1}=\sum_{i=1}^{m} \tilde{P}_{i j} M_{i} V_{i}^{N}, \quad N \geq 0
$$

However, we observe now that (74) is an iterations scheme for the fixed point equation (7). Thus, the results of the previous sections imply that the following limits exist:

$$
\lim _{N \rightarrow \infty} V_{i}^{N}=V_{i}^{*} \quad \text { for every } 1 \leq i \leq m
$$

where $V^{*}=\left(V_{1}^{*}, \ldots, V_{m}^{*}\right)$ is the unique solution of (7). As a consequence of this discussion we have the following:

Theorem 9: The conclusions of theorems 5 and 6 hold true when we replace assumption 2 there by assumption 3 .

\section{Conclusions}

In this paper we consider the dynamics of AIMD networks that evolve according to Markovian dynamics. We have shown that such networks have well-defined stochastic equilibria and provide tools that can be used to characterise these equilibria. In particular, for routers that operate according to assumption 2, we have developed tools for computing $\lim _{k \rightarrow \infty} E(W(k))$ and $\lim _{k \rightarrow \infty} \operatorname{Var}(W(k))$. We then extended these results to the $R$-model given by assumption 3.

Results presented here are concerned with long flows. Although most flows in the Internet are short, the majority of Internet traffic is generated by long flows. Measurements in [26] indicate that for a typical backbone link, $5 \%$ of long flows give rise to $70-85 \%$ of all transferred bytes. Whereas very short flows usually do not have time to react in response to a congestion signal, and in addition it is very hard to predict their behaviour in terms of resource allocation, it will be nevertheless interesting, as a topic for further research, to characterise the behaviour of medium-sized AIMD users. One possible approach for this problem is given in [27].

While developing these tools represents an important first step in studying such networks, much work remains to be done. The results derived in this paper provide tools to address the problem of designing routers that achieve, in the long run, certain goals. By controlling the distribution of the random variable $A(0)$ in the i.i.d. case (1), or the transition matrix $P$ in the Markov cases 2 and 3 , one can guarantee that, in the long run, the asymptotic expected value of $W(k)$ is close to a certain prescribed vector $V^{*}$. A major objective of future work will be to investigate how this might in fact be achieved.

Another interesting designing problem that is of great practical interest, and which may be addressed in the setting provided by either assumption 2 or assumption 3 , is the following. For a prescribed vector $V^{*}$, consider all the transition matrices $P$ for which

$$
\lim _{k \rightarrow \infty} E(W(k))=V^{*}
$$

and among them pick one for which

$$
\lim _{k \rightarrow \infty} \operatorname{Var}(W(k))=T^{*}
$$

is the smallest possible in a certain sense. Minimising the variance makes it more likely that the desired long-run behaviour expressed by $\lim _{k \rightarrow \infty} E(W(k))=V^{*}$ will be realised faithfully (although the cost of this choice may be a slow network convergence or some other undesirable network behaviour). This goal defines a constrained optimisation problem that may be addressed either numerically or theoretically. We note that the minimisation may be approached in various ways: either minimising a certain functional, such as the trace of $T^{*}$, or looking for a $T_{0}^{*}$ such that

$$
T_{0}^{*} \leq T^{*}
$$

in the positive definite sense, where $T_{0}^{*}$ and $T^{*}$ correspond to certain matrices $P_{0}$ and $P$ such that (76) and (77) hold. Finally, we note that one of the principal tools for analysing AIMD networks is the network simulator NS-2. For networks of low dimension, this tool is effective for examining the behaviour of AIMD networks. However, for networks with large number of sources this tool becomes increasingly difficult to use due to excessive simulation times. In this context, efficient methods to compute important network properties are likely of great value to network designers. The tools presented in this paper represent a first step toward the development of such tools.

\section{Acknowledgements}

R. Stanojević and R. Shorten were supported by SFI grant 04/IN1/I1478.

\section{References}

1 Chait, Y., Hollot, C.V., Misra, V.H, Han, H, and Halevi, Y.: 'Dynamic analysis of congested TCP networks'. Proc. American Control Conference, Anchorage, AK, USA, June 1999, pp. 2430 2435

2 Hollot, C.V., and Chait, Y.: 'Non-linear stability analysis of a class of TCP/AQM networks'. Proc. IEEE Conference on Decision and Control, Orlando, FL, USA, December 2001, pp. 2309-2314

3 Johari, R., and Tan, D.: 'End-to-end congestion control for the Internet: delays and stability', IEEE/ACM Trans. Networking, 2001, 9, (6), pp. 818-832

4 Kelly, F.P.: 'Mathematical modelling of the Internet'. Proc. ICIAM 99, 4th International Congress of Industrial Applied Mathematics, Edinburgh, UK, July 1999, pp. 105-116

5 Kunniyur, S.S., and Srikant, R.: 'Stable, scalable, fair congestion control and AQM schemes that achieve high utilisation in the Internet', IEEE Trans. Autom. Control, 2003, 48, (11), pp. 2024-2029

6 Low, S., Paganini, F., and Doyle, J.: 'Internet congestion control', IEEE Control Syst. Mag., 2002, 32, (1), pp. 28-43

7 Mascolo, S.: 'Congestion control in high speed communication networks using the Smith principle', Automatica, 1999, 35, pp. 1921-1935

8 Massoulie, L.: 'Stability of distributed congestion control with heterogeneous feedback delays', IEEE Trans. Autom. Control, 2002, 47, (6), pp. 895-902

9 Vinnicombe, G.: 'On the stability of networks operating TCP-like congestion control'. Cambridge University Statistical Laboratory Research Report, 2000-398, 2000

10 Shorten, R., Wirth, F., and Leith, D.: 'A positive systems model of TCP-like congestion control: asymptotic results', IEEE/ACM Trans. Networking, 2004 (accepted)

11 Zhao, Z., Darbha, S., and Reddy, A.L.N.: 'A method for estimating the proportion of nonresponsive traffic at a router', IEEE/ACM Trans. Networking, 2004, 12, (4), pp. 708-718

12 Jacobson, V.: 'Congestion avoidance and control'. Proc. ACM SIGCOMM '88, Stanford, CA, USA, August 1988, pp. 314-329

13 Jin, S., Guo, L., Matta, I., and Bestavros, A.: 'A spectrum of TCPfriendly window-based congestion control algorithms', IEEE/ACM Trans. Networking, 2003, 11, (3), pp. 341-355

14 Yang, Y., and Lam, S.: 'General AIMD congestion control'. Proc. IEEE International Conference on Network Protocols, Osaka, Japan, November 2000

15 Srikant, R.: 'Internet congestion control' in 'Control theory' (Birkhäuser Boston Inc., Boston, MA, 2004), p. 14

16 Khalifa, I., and Trajkovic, L.: 'An overview and comparison of analytical TCP models'. Proc. 2004 International Symposium on Circuits and Systems, 2004, ISCAS '04, 23-26 May 2004, Vol. 5, pp. $469-472$ 
17 Bacelli, F., and Hong, D.: 'AIMD fairness and fractal scaling of TCP traffic'. Proc. IEEE INFOCOM, New York, NY, USA, June 2002, pp. $229-238$

18 Bacelli, F., and Hong, D.: 'Interaction of TCP flows as billiards'. INRIA Rocquencourt, INRIA Technical Report 4437, 2002

19 Berman, A., and Plemmons, R.: 'Nonnegative matrices in the mathematical sciences', SIAM, 1979

20 Appenzeller, G., Keslassy, I., and McKeown, N.: 'Sizing router buffers'. Proc. ACM SIGCOMM '04, Portland, OR, USA, August/ September 2004, pp. 281-292

21 Qiu, L., Zhang, Y., and Keshav, S.: 'Understanding the performance of many TCP flows', Comput. Networks, 2001, 37, (3-4), pp. 277-306

22 Norris, J.R.: 'Markov chains' (Cambridge University Press, 1997)
23 Wirth, F., Stanojevic, R., Shorten, R., and Leith, D.: 'Stochastic equilibria of AIMD communication networks', SIAM J. Matrix Anal. Appl., 2005 (accepted for publication)

24 Hartfiel, D.: 'Nonhomogeneous matrix products', World Scientific, 2002

25 Elsner, L., Koltracht, I., and Neumann, M.: 'On the convergence of asynchromous paracontractions with applications to tomographic reconstruction from incomplete data', Linear Algebra Appl., 1990, 130, pp. 65-82

26 Estan, C., and Varghese, G.: 'New directions in traffic measurement and accounting'. Proc. ACM SIGCOMM'02, Pittsburgh, PA, August 2002, pp. 323-336

27 Hespanha, J.P.: 'Stochastic hybrid systems: Applications to communication networks' in Alur, R., and Pappas, G.J. (Eds.): 'Hybrid systems: computation and control', number 2993 (LNCS, 2004) 